\title{
Weighted voting procedure having a unique blocker
}

\author{
Sanjay Bhattacherjee ${ }^{1,2}$ (D) Palash Sarkar ${ }^{3}$ (i)
}

Accepted: 1 December 2020 / Published online: 7 January 2021

(c) The Author(s) 2020

\begin{abstract}
The Goods and Services Tax (GST) Council of India has a non-conventional weighted voting procedure having a primary player who is a blocker and a set of secondary players. The voting weights are not fixed and are determined based on the subset of players which participate in the voting. We introduce the notion of voting schema to formally model such a voting procedure. Individual voting games arise from a voting schema depending on the subset of secondary players who participate in the voting. We make a detailed formal study of the trade-off between the minimal sizes of winning and blocking coalitions in the voting games that can arise from a voting schema. Finally, the GST voting procedure is assessed using the theoretical results leading to suggestions for improvement.
\end{abstract}

Keywords Goods and Services Tax (GST) · GST Council • Voting schema • Weighted majority voting games · Winning and blocking coalition · Blocking power

JEL Classification C7 - D7 · H1 - H2 $\cdot$ H7

\section{Introduction}

Taxation policy is a crucial factor in determining the overall health of the economy of a nation and the well being of its citizens. Determining taxation policy is a political process. India is a multi-party democracy with a federal structure consisting of a Union government (called the Centre) and provincial governments (called the States).

Sanjay Bhattacherjee

sanjay.bhattacherjee@gmail.com

Palash Sarkar

palash@isical.ac.in

1 Turing Lab, Applied Statistics Unit, Indian Statistical Institute, 203, B. T. Road, Kolkata 700108, India

2 Present Address: School of Computing, University of Kent, CT2 7NF Kent, UK

3 Applied Statistics Unit, Indian Statistical Institute, 203, B. T. Road, Kolkata 700108, India 
Achieving a stable taxation policy requires the participation of the relevant political parties governing the Centre and the States.

On 1st July 2017, a landmark taxation law came into effect in India through the implementation of the Constitution (One Hundred and First Amendment) Act, 2016 which replaced the multitude of existing taxes by a single Goods and Services Tax (GST). ${ }^{1}$ The new act inserts Article 279A into the Constitution of India. This Article requires the creation of a constitutional body called the GST Council. Among other things, Article 279A specifies the decision making mechanism to be followed in the GST Council.

Voting procedure in the GST council Clause 9 of Article 279A specifies the following.

"Every decision of the Goods and Services Tax Council shall be taken at a meeting, by a majority of not less than three-fourths of the weighted votes of the members present and voting, in accordance with the following principles, namely:

(a) the vote of the Central Government shall have a weightage of one-third of the total votes cast, and

(b) the votes of all the State Governments taken together shall have a weightage of two-thirds of the total votes cast, in that meeting."

From the above, it becomes clear that the voting procedure in the GST Council is a weighted voting mechanism. The purpose of this work is to analyse the GST voting rules using the formal framework of voting games.

There is a rich and several decades old literature on voting games. The notion of voting power of a player was proposed by Penrose (1946) and later by Banzhaf (1965). Shapley (1953) and Shapley and Shubik (1954) studied voting in the framework where the order in which votes are cast is important. There has been a great deal of work on formal analysis of voting games. We refer the reader to Felsenthal and Machover (1998) and Laruelle and Valenciano (2011) for details and the surveys (Napel 2016; Kurz et al. 2015) for discussions on the various topics in the area. A textbook level introduction to the area can be found in Chakravarty et al. (2015).

Most of the literature on voting games has studied the setting where each player has two options (to vote 'yes', or to vote 'no') and the outcome is binary. Laruelle and Valenciano (2012) studied the setting where each player has four choices, namely to vote 'yes', to vote 'no', to abstain, or to be absent and the outcome is still binary. The more general framework where the players have multiple options and and there could also be more than two outcomes has been analysed by Freixas and Zwicker (2003) and Freixas and Zwicker (2009).

Use of weighted voting for taking decision is employed in various public bodies. Two of the most important examples are the International Monetary Fund (IMF) and the European Union (EU). Analysis of the voting procedures in these bodies using the formalism of voting games have been reported in the literature (Leech 2002a, b).

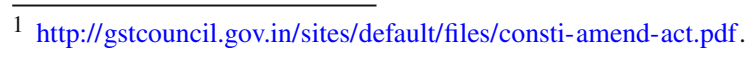


Other examples include elections in the Senate of US, and in the parliaments of several European countries.

The voting rules of the GST Council do not specify a single voting game. Since the number of members "present and voting" can vary, a number of voting games can arise. Further, the voting weights of the members are not fixed. Rather, it is only mentioned that "the vote of the Central Government shall have a weightage of one-third of the total votes cast". The rules do not specify how the rest two-third of the total voting weight are to be distributed among other members. However, a resolution can not pass without the Centre. So, every voting game includes at least one player - the Centre.

We introduce a new notion, which we call voting schema, to formally model the voting rules in the GST Council. In a voting schema, there is a primary player and a set of secondary players. The primary player represents the Centre, while the secondary players represent the States. In a particular meeting, a set of secondary players may be present and voting. So, a voting schema gives rise to a number of voting games, one for each set of secondary players who participate in the voting.

We assume that the secondary players have some a priori weights. Depending on the actual set of players who participate in the voting, the voting weights need to be determined. The primary player will obtain a fraction $\lambda$ of the total voting weight while the other $(1-\lambda)$ fraction of the voting weight will be distributed among the secondary players "present and voting" in proportion in proportion to their a priori weights. A method for obtaining such voting weights is described and proved to be essentially unique.

We make a detailed formal study of voting games arising from voting schemas of the above type. The primary player's voting weight is such that it can block any resolution, but, will need the support of some secondary players to pass a resolution. A set of secondary players, on the other hand, cannot pass a resolution without the primary player, but may be able to block a resolution. This tension between the primary player's ability to pass a resolution and a group of secondary players' ability to block a resolution is formally modelled as $(\alpha, \beta)$-efficiency of the game, where $\alpha$ is the minimum number of secondary players required by the primary player to pass a resolution and $\beta$ is the minimum number of secondary players required to block a resolution.

Games arising from the condition that the a priori weights of the secondary players are equal are called uniform games. We prove that the values of $\alpha$ and $\beta$ are maximised if the game is uniform. Further, in a uniform game, if $n$ players participate in the voting, then $\alpha+\beta=n+1$. The combination of these two results show that for any game which is $(\alpha, \beta)$-efficient, the condition $\alpha+\beta \leq n+1$ must hold.

We argue that the weight of the primary player should be such that it is a blocker, but no more. To achieve this, we consider games with modified winning condition (in short, modified games), where the winning condition is changed from " $\geq$ " to " $>$ ", and where the winning threshold $q$ equals $1-\lambda$. Analogues of the results on $(\alpha, \beta)$-efficiency mentioned above are stated for modified games.

The final section transfers the theoretical results back to the context of GST voting rules. A set of four desiderata are derived, namely, the Center should be a blocker, its weight should be sufficient to ensure it is a blocker and no more, the weight of any State should be less than that of the Centre, and the Centre's task of mustering 
a passing coalition should be at least as difficult as the task of a group of States to block a resolution moved by the Centre. It is shown that the present voting rules in the GST Council satisfy the first and the fourth desiderata, but not the second and the third desiderata. We propose some modifications to the GST voting rules such that all the four desiderata are satisfied. The rules arising from the suggested modifications are quite close to the present GST voting rules and can be easily incorporated into the GST Act.

\section{Background on voting games}

The following notation will be used.

- The cardinality of a finite set $S$ will be denoted by $\# S$.

- For a real number $x,\lfloor x\rfloor$ will denote the greatest integer not greater than $x$ and $\lceil x\rceil$ will denote the least integer not lesser than $x$.

- The greatest common divisor of the integers $a_{1}, \ldots, a_{n}$ will be denoted by $\operatorname{gcd}\left(a_{1}, \ldots, a_{n}\right)$.

We provide some standard definitions arising in the context of voting games. For details the reader may consult (Felsenthal and Machover 1998; Chakravarty et al. 2015).

Let $N=\left\{A_{1}, A_{2}, \ldots, A_{n}\right\}$ be a set of $n$ players. A subset of $N$ is called a voting coalition. The set of all voting coalitions is denoted by $2^{N}$. A voting game $G$ is given by its characteristic function $\chi_{G}: 2^{N} \rightarrow\{0,1\}$ where a winning coalition is assigned the value 1 and a losing coalition is assigned the value 0 . For a voting game $G$, the set of all winning coalitions will be denoted by $W(G)$ and the set of all losing coalitions will be denoted by $L(G)$. Below we recall some basic notions about voting games.

1. A voting game $G$ is said to be monotone if whenever $S$ is a winning coalition which is contained in a coalition $T$, then $T$ is also a winning coalition.

2. A voting game $G$ is said to be proper if for any coalition $S \subseteq N, \chi_{G}(S)=1$ implies that $\chi_{G}(N \backslash S)=0$. In other words, in a proper game it is not allowed for both $S$ and its complement to be winning.

3. A coalition $S \subseteq N$ is called a minimal winning coalition if $\chi_{G}(S)=1$ and there is no $T \subset S$ for which $\chi_{G}(T)=1$.

4. A player $A_{i}$ is said to be the dictator in $G$, if $\left\{A_{i}\right\}$ is the only minimal winning coalition in $G$.

5. A coalition $S \subseteq N$ is called a blocking coalition if $\chi_{G}(N \backslash S)=0$. If $A_{i}$ is a player such that $\left\{A_{i}\right\}$ is a blocking coalition, then $A_{i}$ is said to be a blocker.

6. A blocking coalition $S$ is said to be a minimal blocking coalition if for any $A_{i} \in S$, $S \backslash\left\{A_{i}\right\}$ is not a blocking coalition. In other words, if any player is dropped from $S$, then $S$ no longer remains a blocking coalition.

Definition 1 (Weighted majority voting game) Consider a triplet $(N, \mathbf{w}, q)$, where $N=\left\{A_{1}, \ldots, A_{n}\right\}$ is a set of players; $\mathbf{w}=\left(w_{1}, w_{2}, \ldots, w_{n}\right)$ is a vector of nonnegative weights with $w_{i}$ being the weight of $A_{i}$; and $q$ is a real number in $(0,1)$. Let $\omega=\sum_{i=1}^{n} w_{i}$. The triplet $(N, \mathbf{w}, q)$ defines a weighted majority voting game $G$ 
given by its characteristic function $\chi_{G}: 2^{N} \rightarrow\{0,1\}$ in the following manner. Let $w_{S}=\sum_{A_{i} \in S} w_{i}$ denote the sum of the weights of all the players in the coalition $S \subseteq N$. Then

$$
\chi_{G}(S)= \begin{cases}1 & \text { if } w_{S} / \omega \geq q, \\ 0 & \text { otherwise }\end{cases}
$$

We will write $G=(N, \mathbf{w}, q)$ to denote the weighted majority voting game arising from the triplet $(N, \mathbf{w}, q)$. The quantity $q$ is called the winning threshold.

Suppose, we have a triplet $(N, \mathbf{w}, q)$ as in Definition 1 , but, the winning condition in (1) is changed from " $\geq$ " to " $>$ ". This also gives rise to a voting game. By $\widetilde{G}=$ $(N, \mathbf{w}, q)$, we denote the voting game whose characteristic function is the following.

$$
\chi_{\widetilde{G}}(S)= \begin{cases}1 & \text { if } w_{S} / \omega>q \\ 0 & \text { otherwise }\end{cases}
$$

The game $\widetilde{G}=(N, \mathbf{w}, q)$ will be called a modified weighted majority voting game (or, simply a modified game).

\subsection{Efficiency of a game with respect to a blocker}

Suppose a game has at least one blocker and fix one such blocker. The blocker can block the passing of any resolution, but, need not be able to ensure by itself that a resolution passes. So, for passing a resolution, a blocker would require support of other players. On the other hand, it is also of interest to know which other coalitions not containing the blocker can prevent a resolution from being passed. These considerations motivate us to define the following notion.

Definition 2 Suppose $G$ is a voting game on a set of players $N=\left\{A_{0}, A_{1}, \ldots, A_{n}\right\}$ and let $A_{0}$ be a blocker. The game $G$ is said to be $(\alpha, \beta)$-efficient with respect to $A_{0}$ if the following two conditions hold.

- Any minimal winning coalition containing $A_{0}$ contains at least $\alpha$ players other than $A_{0}$.

- Any minimal blocking coalition not containing $A_{0}$ contains at least $\beta$ players.

\section{Voting schema}

The GST voting procedure essentially gives rise to a voting schema rather than a single voting game. There is a set of players corresponding to the members of the council and various games arise depending upon the players that are "present and voting". We formalise the notion of voting schema below. 
Definition 3 A voting schema $\mathcal{S}$ is a tuple $(m, \mathbf{v}, q, \lambda, \tau)$, where $m$ is a positive integer, $\mathbf{v}=\left(v_{1}, \ldots, v_{m}\right)$ is a set of non-negative integers, $q \in(1 / 2,1)$, $\lambda$ is a rational number in $(0, q)$, and $\tau \in(0,1)$.

A voting schema $\mathcal{S}=(m, \mathbf{v}, q, \lambda, \tau)$ implicitly defines the set of players $\left\{A_{0}, A_{1}, \ldots, A_{m}\right\}$. The player $A_{0}$ is the primary player while all the other players are secondary players. The value $v_{i}$ is the a priori weight of player $A_{i}$. A number of voting games can arise from $\mathcal{S}$ by specifying the set of players to be $N=\left\{A_{0}\right\} \cup V$ where $V$ is a subset of $\left\{A_{1}, \ldots, A_{m}\right\}$ satisfying $\# V \geq \tau m$. We consider $\tau$ to be a voting quorum, i.e., a minimum of $\tau m$ secondary players must participate in the voting for the voting to be considered valid. Let $\# V=n$. This gives rise to a weighted majority voting game $G$ consisting of the $(n+1)$ players in $N$. The winning threshold in $G$ is given by $q$. Suppose the sum of the voting weights of all the $(n+1)$ players in $G$ is $\omega$. The voting weight of the primary player $A_{0}$ is a fraction $\lambda$ of $\omega$ and the combined voting weights of all the secondary players is a fraction $1-\lambda$ of $\omega$. Further, the voting weights of the secondary players in $G$ are proportional to the corresponding entries of the players in the vector $\mathbf{v}$.

\subsection{Determining voting weights}

Consider a voting schema $\mathcal{S}=(m, \mathbf{v}, q, \lambda, \tau)$. Suppose a game $G$ is formed with the player set $N=\left\{A_{0}\right\} \cup V$ where $V=\left\{A_{j_{1}}, \ldots, A_{j_{n}}\right\} \subseteq\left\{A_{1}, \ldots, A_{m}\right\}$ and $\# V \geq \tau m$. For $i=1, \ldots, n$, let $u_{i}=v_{j_{i}}$. In $G$, the primary player $A_{0}$ is to have a $\lambda$ fraction of the total voting weight. The requirement is to distribute the other $1-\lambda$ fraction of the total voting weight to the secondary players in $V$ in proportion to the a priori weights $u_{1}, \ldots, u_{n}$. A technique is required to come up with weights $w_{0}, w_{1}, \ldots, w_{n}$ such that $w_{0}$ is the weight of the primary player and $w_{1}, \ldots, w_{n}$ are the weights of the secondary players satisfying the required conditions. The following result provides such a technique.

Theorem 1 Let $u_{1}, \ldots, u_{n}$ be positive integers and $\lambda=a / b \in(0,1)$ where $a$ and $b$ are positive integers. Let

$$
w_{0}=\frac{u a}{d}, \quad w_{i}=\frac{u_{i}(b-a)}{d} \quad i=1, \ldots, n
$$

where $u=u_{1}+\cdots+u_{n}$ and $d=\operatorname{gcd}\left(u a, u_{1}(b-a), \ldots, u_{n}(b-a)\right)$. Then $w_{0}, w_{1}, \ldots, w_{n}$ satisfy

$$
\frac{w_{0}}{\omega}=\lambda, \frac{w_{i}}{\omega}=(1-\lambda) \frac{u_{i}}{u}
$$

where $\omega=w_{0}+w_{1}+\cdots+w_{n}$.

Further, if $w_{0}^{\prime}, w_{1}^{\prime}, \ldots, w_{n}^{\prime}$ are integer values such that $w_{0}^{\prime} / \omega^{\prime}=\lambda$ and $w_{i}^{\prime} / \omega^{\prime}=$ $(1-\lambda) u_{i} / u$ for $i=1, \ldots, n$, where $\omega^{\prime}=w_{0}^{\prime}+w_{1}^{\prime}+\cdots+w_{n}^{\prime}$, then $w_{j}^{\prime}=\mu w_{j}$ for $j=0, \ldots, n$ for some non-zero integer $\mu$. 
Equation (3) provides the weights that are to be assigned to the primary player and the secondary players. Equation (4) shows the correctness of these weights, i.e., the primary player obtains a fraction $\lambda$ of the total weight and the other $1-\lambda$ fraction is distributed among the secondary players in proportion to their a priori weights. The second part of Theorem 1 shows that the weights obtained in (3) are unique up to scaling by a non-zero integer.

Corollary 1 Let $u_{1}, \ldots, u_{n}$ and $u_{1}^{\prime}, \ldots, u_{n}^{\prime}$ be positive integers such that $u_{i}^{\prime}=v u_{i}$ for some non-zero integer $v$. Let $w_{0}, w_{1}, \ldots, w_{n}$ be obtained from $u_{1}, \ldots, u_{n}$ as in (3) and $w_{0}^{\prime}, w_{1}^{\prime}, \ldots, w_{n}^{\prime}$ be obtained from $u_{1}^{\prime}, \ldots, u_{n}^{\prime}$ as in (3). Then $w_{i}=w_{i}^{\prime}$ for $i=0,1, \ldots, n$.

The interpretation of Corollary 1 is that scaling of the a priori weights of the secondary players does not change the eventual weights assigned to the primary player and the secondary players.

\section{Voting games from a voting schema}

We formally introduce the voting games which can arise from a voting schema.

Definition 4 Let $\mathcal{S}=(m, \mathbf{v}, q, \lambda, \tau)$ be a voting schema, with $\mathbf{v}=\left(v_{1}, \ldots, v_{m}\right)$ and $\lambda=a / b$. This implicitly gives rise to the primary player $A_{0}$ and the set of secondary players $\left\{A_{1}, \ldots, A_{m}\right\}$, where the a priori weight of the secondary player $A_{i}$ is $v_{i}$. Let $\tau m \leq n \leq m$ and suppose the set of secondary players $V=\left\{A_{j_{1}}, \ldots, A_{j_{n}}\right\}$ participate in the voting. Let $u_{1}=v_{j_{1}}, \ldots, u_{n}=v_{j_{n}}$ and $\mathbf{u}=\left(u_{1}, \ldots, u_{n}\right)$. The game $G_{m, n, \mathbf{v}, V, q, \lambda, \tau}$ is defined to be the weighted majority voting game on $n+1$ players as follows.

$$
G_{m, n, \mathbf{v}, V, q, \lambda, \tau}=\left(\left\{A_{0}\right\} \cup V, \mathbf{w}, q\right)
$$

where $\mathbf{w}=\left(w_{0}, w_{1}, \ldots, w_{n}\right) ; w_{0}$ is the weight of $A_{0} ;$ and $w_{1}, \ldots, w_{n}$ are the weights of $A_{j_{1}}, \ldots, A_{j_{n}}$ respectively. The components of $\mathbf{w}$ are obtained from the components of $\mathbf{u}$ as given by (3).

When the parameters $m, n, \mathbf{v}, V, q, \lambda$ and $\tau$ are understood from the context, we will write $G$ instead of $G_{m, n, \mathbf{v}}, V, q, \lambda, \tau$.

\section{Remarks}

1. The condition $q \in(1 / 2,1)$ ensures that the game is proper.

2. The upper bound $\lambda<q$ ensures that the primary player is not a dictator and cannot pass a resolution by itself.

3. The primary player $A_{0}$ is a blocker if and only if $q>1-\lambda$.

4. Consider a winning coalition consisting of the primary player and a coalition of the secondary players whose weight is a fraction $p$ of the total voting weights of the secondary players. Then $\lambda+(1-\lambda) p \geq q$ and so $p \geq(q-\lambda) /(1-\lambda)$. If the primary player is a blocker, i.e., $q>1-\lambda$, then instead of $G_{m, n, \mathbf{v}, V, q, \lambda, \tau}$, one 
may study the game which consists only of the secondary players (leaving out the primary player) with the winning threshold being $(q-\lambda) /(1-\lambda)$. This approach, however, does not reduce any of the complexity of the original set-up. The values of $q$ and $\lambda$ still need to be specified and the technique for determining the voting weights of the secondary players is still required. On the contrary, this approach loses generality in a priori requiring the primary player to be a blocker.

The primary player has a special status. The requirement that the weight of the primary player should be more than that of any of the secondary players is characterised in the following result.

Proposition 1 Considera game $G_{m, n, \mathbf{v}, V, q, \lambda, \tau}$ with $V=\left\{A_{j_{1}}, \ldots, A_{j_{n}}\right\}$ and $u_{i}=v_{j_{i}}$, $i=1, \ldots, n$. The secondary player $A_{j_{i}}$ has voting weight less than that of the primary player $A_{0}$, i.e., $w_{i}<w_{0}$ if and only if

$$
\frac{u_{i}}{u_{1}+\cdots+u_{n}}<\frac{\lambda}{1-\lambda} .
$$

Suppose $\lambda=1 / 3$ and $u_{1}=\cdots=u_{n}$. From Proposition 1 , a secondary player has voting weight less than the primary player if and only if $n>2$. So, apart from the primary player, if there are at most two secondary players in the game, then the voting weights of the secondary players will be greater than that of the primary player. The threshold of voting quorum $\tau$ can be used to ensure that a certain minimum number of secondary players are present in the game which would rule out such anomalies.

Proposition 2 Suppose a game $G_{m, n, \mathbf{v}, V, q, \lambda, \tau}$ is $(\alpha, \beta)$-efficient with respect to the primary player. Then $\alpha<\beta$ if and only if $q \leq(\lambda+1) / 2$.

\subsection{Uniform games}

Of special interest is the case when the a priori weights of all the secondary players are equal.

Definition 5 A voting schema $\mathcal{S}=(m, \mathbf{v}, q, \lambda, \tau)$ is said to be uniform if $v_{1}=\cdots=$ $v_{m}$. A voting game $G_{m, n, \mathbf{v}}, V, q, \lambda, \tau$ arising from a uniform voting schema will also be called uniform.

In view of Corollary 1 , in a uniform game we may take $v_{1}=\cdots=v_{m}=1$ and consequently from (3), $w_{1}=\cdots=w_{n}=(b-a) / d$ where $\lambda=a / b$ and $d=$ $\operatorname{gcd}(n a,(b-a))$.

The importance of such games arises from the following result.

Theorem 2 Let $\mathbf{v}=\left(v_{1}, \ldots, v_{m}\right)=(1, \ldots, 1)$ and $\mathbf{v}^{\prime}=\left(v_{1}^{\prime}, \ldots, v_{m}^{\prime}\right)$ where not all the $v_{i}^{\prime}$ 's are equal. Suppose a game $G_{m, n, \mathbf{v}, V, q, \lambda, \tau}$ is $(\alpha, \beta)$-efficient with respect to the primary player and a game $G_{m, n, \mathbf{v}^{\prime}, V, q, \lambda, \tau}^{\prime}$ is $\left(\alpha^{\prime}, \beta^{\prime}\right)$-efficient with respect to the primary player. Then $\alpha \geq \alpha^{\prime}$ and $\beta \geq \beta^{\prime}$.

In other words, we have the following. Let $G_{m, n, \mathbf{v}, V, q, \lambda, \tau}$ be a game which is $(\alpha, \beta)$ efficient with respect to the primary player. Theorem 2 shows that if $G_{m, n, \mathbf{v}, V, q, \lambda, \tau}$ is uniform, then $\alpha$ and $\beta$ take the maximum possible values. 
We next consider the possible values of $\alpha$ and $\beta$ in a uniform game which is $(\alpha, \beta)$ efficient with respect to the primary player. One may wish to increase the values of both $\alpha$ and $\beta$ by appropriately setting the values of $\lambda$ and $q$. The next result shows that this is not possible.

Theorem 3 A uniform game $G_{m, n, \mathbf{v}, V, q, \lambda, \tau}$ is $(\mathfrak{p}, \mathfrak{q})$-efficient with respect to the primary player where $\mathfrak{p}=n-\rho$ and $\mathfrak{q}=1+\rho$ with $\rho=\left\lfloor\frac{n(1-q)}{(1-\lambda)}\right\rfloor$. Consequently, $\mathfrak{p}+\mathfrak{q}=n+1$.

There are several consequences of Theorem 3.

1. For fixed $q$ and $\lambda$, both $\mathfrak{p}$ and $\mathfrak{q}$ decrease with decreasing $n$. So, as the number of secondary players which participate in voting goes down, the primary player requires lesser number of secondary players to win and also a lesser number of secondary players can form a blocking coalition.

2. For fixed $q$ and $n$, as $\lambda$ increases, the value of $\rho$ increases in unit steps from $n(1-q)$ to $2 n(1-q)-1$.

Combining Theorems 2 and 3 , we obtain the following result.

Theorem 4 Suppose a game $G_{m, n, \mathbf{v}, V, q, \lambda, \tau}$ (which is not necessarily uniform) is $(\alpha, \beta)$-efficient with respect to the primary player. Then $\alpha+\beta \leq n+1$.

Suppose that $q>1-\lambda$ so that the primary player is a blocker. In this setting, for fixed $n$ and $q$, it is of interest to determine the effect of changing $\lambda$ on $\mathfrak{p}$ and $\mathfrak{q}$.

Theorem 5 Let $G_{m, n, \mathbf{v}, V, q, \lambda_{1}, \tau}$ be a uniform game with $q>1-\lambda_{1}$ which is $\left(\mathfrak{p}_{1}, \mathfrak{q}_{1}\right)$ efficient with respect to the primary player and let $G_{m, n, \mathbf{v}}, V, q, \lambda_{2}, \tau$ be a uniform game with $q>1-\lambda_{2}$ which is $\left(\mathfrak{p}_{2}, \mathfrak{q}_{2}\right)$-efficient with respect to the primary player. Then

1. $\lambda_{1} \geq \lambda_{2}$ if and only if $\mathfrak{p}_{1} \leq \mathfrak{p}_{2}$.

2. $\lambda_{1} \geq \lambda_{2}$ if and only if $\mathfrak{q}_{1} \geq \mathfrak{q}_{2}$.

\section{Modified voting games from a voting schema}

Suppose that we wish to determine the minimum value of $\lambda$ such that the primary player is a blocker. In the game $G_{m, n, \mathbf{v}, V, q, \lambda, \tau}$, any value of $\lambda$ and $q$ such that $\lambda>1-q$ will ensure that the primary player is a blocker. This creates a problem. The condition $\lambda$ greater than $1-q$ does not provide a unique value of $\lambda$. For any choice $\lambda_{1}$ of $\lambda$, it is possible to find a $\lambda_{2}$ such that $1-q<\lambda_{2}<\lambda_{1}$. So, the problem of assigning the minimum possible weight to the primary player resulting in it being a blocker cannot be solved.

To tackle this problem, we consider modified weighted majority voting games whose winning condition is given by (2). We make the following definition.

Definition 6 Let $\mathcal{S}=(m, \mathbf{v}, q, \lambda, \tau)$ be a voting schema, with $\mathbf{v}=\left(v_{1}, \ldots, v_{m}\right)$, $\lambda=a / b$ and $q=1-\lambda$. This implicitly gives rise to the primary player $A_{0}$ and the set of secondary players $\left\{A_{1}, \ldots, A_{m}\right\}$, where the a priori weight of the secondary player $A_{i}$ is $v_{i}$. Let $\tau m \leq n \leq m$ and suppose the set of secondary players $V=\left\{A_{j_{1}}, \ldots, A_{j_{n}}\right\}$ 
participate in the voting. Let $u_{1}=v_{j_{1}}, \ldots, u_{n}=v_{j_{n}}$ and $\mathbf{u}=\left(u_{1}, \ldots, u_{n}\right)$. The game $\widetilde{G}_{m, n, \mathbf{v}, V, \lambda, \tau}$ is defined to be a modified weighted majority voting game on $n+1$ players as follows.

$$
\widetilde{G}_{m, n, \mathbf{v}, V, \lambda, \tau}=\left(\left\{A_{0}\right\} \cup V, \mathbf{w}, q\right)
$$

where $\mathbf{w}=\left(w_{0}, w_{1}, \ldots, w_{n}\right) ; w_{0}$ is the weight of $A_{0}$; and $w_{1}, \ldots, w_{n}$ are the weights of $A_{j_{1}}, \ldots, A_{j_{n}}$ respectively. The components of $\mathbf{w}$ are obtained from the components of $\mathbf{u}$ as given by (3).

\section{Remarks}

1. In the voting schema $\mathcal{S}=(m, \mathbf{v}, q, \lambda, \tau)$ and a modified voting game $G_{m, n, \mathbf{v}, V, \lambda, \tau}$ arising from $\mathcal{S}$, the value of $q$ is fixed to $1-\lambda$. This ensures that the primary player $A_{0}$ is a blocker and further, has the minimum possible weight which makes it a blocker.

2. For the game to be proper, we require $q>1 / 2$ which combined with $q=1-\lambda$ implies that $\lambda<1 / 2$. Since $q>1 / 2$ and $\lambda<1 / 2$, it follows that $\lambda<q$ and so the primary player is not a dictator.

We observe that Proposition 1 characterises the condition that the secondary players have voting weights less than that of the primary player. This result does not require the winning condition. So, Proposition 1 also holds for modified games.

Suppose $\mathcal{S}=(m, \mathbf{v}, 1-\lambda, \lambda, \tau)$ is a uniform voting schema, i.e., $v_{1}=\cdots=v_{n}$. A modified voting game $\widetilde{G}_{m, n, \mathbf{v}, V, \lambda, \tau}$ arising from $\mathcal{S}$ will be called a modified uniform voting game. It is possible to derive results on modified uniform voting games which are analogous to results on uniform voting games. These are mentioned below.

Theorem 6 Let $\mathbf{v}=\left(v_{1}, \ldots, v_{m}\right)=(1, \ldots, 1) \underset{\widetilde{G}}{\text { and }} \mathbf{v}^{\prime}=\left(v_{1}^{\prime}, \ldots, v_{m}^{\prime}\right)$ where not all the $v_{i}^{\prime}$ 's are equal. Suppose a modified game $\widetilde{G}_{m, n, \mathbf{v}, V, \lambda, \tau}$ is $(\alpha, \beta)$-efficient with respect to the primary player and a game $\widetilde{G}_{m, n, \mathbf{v}^{\prime}, V, \lambda}^{\prime}$ is $\left(\alpha^{\prime}, \beta^{\prime}\right)$-efficient with respect to the primary player. Then $\alpha \geq \alpha^{\prime}$ and $\beta \geq \beta^{\prime}$.

Theorem 7 A uniform modified game $\widetilde{G}_{m, n, \mathbf{v}}, V, \lambda, \tau$ is $(\mathfrak{s}, \mathfrak{t})$-efficient with respect to the primary player where $\mathfrak{s}=n-\delta+1, \mathfrak{t}=\delta$ and $\delta=\left\lceil\frac{n \lambda}{1-\lambda}\right\rceil$. Consequently, we have $\mathfrak{s}+\mathfrak{t}=n+1$.

Combining Theorems 6 and 7 , we obtain the following result.

Theorem 8 Suppose a modified game $\widetilde{G}_{m, n, \mathbf{v}, V, \lambda, \tau}$ (which is not necessarily uniform) is $(\alpha, \beta)$-efficient with respect to the primary player. Then $\alpha+\beta \leq n+1$.

Theorem 9 Let $\widetilde{G}_{m, n, \mathbf{v}, V, \lambda_{1}, \tau}$ be a uniform modified game which is $\left(\mathfrak{s}_{1}, \mathfrak{t}_{1}\right)$-efficient with respect to the primary player and let $\widetilde{G}_{m, n, \mathbf{v}, V, \lambda_{2}, \tau}$ be a uniform modified game which is $\left(\mathfrak{s}_{2}, \mathfrak{t}_{2}\right)$-efficient with respect to the primary player. Then

1. $\lambda_{1} \geq \lambda_{2}$ if and only if $\mathfrak{s}_{1} \leq \mathfrak{s}_{2}$.

2. $\lambda_{1} \geq \lambda_{2}$ if and only if $\mathfrak{t}_{1} \geq \mathfrak{t}_{2}$.

Proposition 3 Suppose a modified game $\widetilde{G}_{m, n, \mathbf{v}, V, \lambda, \tau}$ is $(\alpha, \beta)$-efficient with respect to the primary player. Then $\alpha<\beta$ if and only if $\lambda>1 / 3$. 


\section{Voting in the GST Council}

In the last few sections, we have studied voting games arising from a voting schema. This has been done in an abstract theoretical set-up. In the present section, we connect the formal analysis to the actual voting procedure in the GST Council. The present voting procedure of the GST Council is captured by a voting schema $\mathcal{G S T}=(m, \mathbf{v}, q, \lambda, \tau)$, where

$-m=31, q=3 / 4$, and $\lambda=1 / 3$;

$-\mathbf{v}$ and $\tau$ are not specified.

Choice of $\mathbf{v}$ Since the voting rules do not specify $\mathbf{v}$, one may consider several possibilities for determining $\mathbf{v}$, namely, the entries of $\mathbf{v}$ are equal, the entries of $\mathbf{v}$ are determined by the sizes of the population of the States, and the entries of $\mathbf{v}$ are determined by the GDP of the States. Whatever be the choice of $\mathbf{v}$, the actual voting weights are to be determined using Theorem 1.

Meeting quorum The GST rules specify a meeting quorum which states that for a meeting to be considered valid more than half of the members must be present. Our formalisation of the GST voting rules does not capture meeting quorum. This is because, given the importance of the GST Council, it is extremely unlikely that a meeting quorum will not be attained.

Voting quorum We have introduced the parameter $\tau$ which represents the threshold of voting quorum. This notion of voting quorum is not part of the GST voting rules. Since the actual voting weights depend on the States which take part in the voting, the notion of voting quorum becomes important in ensuring that the States have voting weights less than that of the Centre (see Proposition 1).

In a federal set-up, there should be checks on the power of the Centre. In the context of the GST Council, these checks come in two forms. On the one hand, the Centre should not be able to pass a resolution with the support of only a small number of States, while, on the other hand, blocking a resolution moved by the Centre should not require a large number of States to form a coalition. With respect to these checks, Theorems 2, 3 and 4 uncover a dichotomous situation. If the a priori weights are made equal, then the Centre's task of mustering a winning coalition becomes more difficult and the task of forming a blocking coalition by the States also becomes more difficult. Conversely, if unequal a priori weights are used, then the States' task of forming a blocking coalition becomes easier and the Centre's task of forming a winning coalition also becomes easier.

Theorem 5 shows that as the fraction of the Centre's weight increases, the Centre requires lesser number of partners to pass a resolution while a greater number of States must come together to block a resolution. Consequently, providing more weight to the Centre decreases its dependence on the States and so works against the principle of decentralisation. 


\subsection{GST voting desiderata}

We list down a set of basic desiderata and their rationale that a voting procedure in the GST Council should satisfy.

Desideratum 1: The Centre should be a blocker. The Centre enjoys the confidence of the Lok Sabha which is the apex legislative body in the country. So, any resolution that is passed should have the approval of the Centre and by implication the approval of the Lok Sabha.

Desideratum 2: The Centre's weight should be enough to ensure it is a blocker and no more. While the Centre should certainly be a blocker, we do not find any socio-political justification to assign more weight to the Centre beyond the minimum necessary to ensure that it is a blocker.

Desideratum 3: The weight of any State should be less than that of the Centre. While any State represents only a segment of the population, the Centre represents the whole population. In view of this, it is reasonable to require that the Centre has weight more than any of the States. Note that Desiderata 2 and 3 together imply that no State is a blocker. ${ }^{2}$

Desideratum 4: The game should be $(\alpha, \beta)$-efficient with respect to the Centre where $\alpha \geq \beta$. This condition will ensure that the Centre will require at least as many States to pass a resolution as the minimum number of States who need to come together to block a resolution. In other words, the Centre's job of mustering a supporting coalition should not be any easier than the possible role of a coalition of States to block a resolution.

Desiderata and the present GST rules Since the Centre is a blocker, Desideratum 1 is satisfied by the present GST rules. On the other hand, as discussed at the start of Sect. 5 , it is not possible to satisfy Desideratum 2 when the winning condition is " $\geq$ ". Since the winning condition in the GST rules is indeed of the type " $\geq$ ", Desideratum 2 is not satisfied by the present GST rules. A characterisation of the condition that the weight of any State is less than that of the Centre is given by Proposition 1. This condition cannot be ensured without specifying a threshold of voting quorum. The present GST rules do not mention voting quorum and so, Desideratum 3 is not ensured to hold. Since the GST Act specifies $\lambda=1 / 3$ and $q=3 / 4$, from Proposition 2 the condition $\alpha \geq \beta$ holds and so Desideratum 4 holds for the present GST rules.

Suggestions for modifying voting rules in the GST Council To satisfy Desiderata 1 and 2, we suggest that a voting schema with $q=1-\lambda$ and modified voting game be adopted. To ensure Desideratum 3, it is required to choose an appropriate value for the voting quorum parameter $\tau$. Suppose $\lambda=1 / 4$. Using Proposition 1 , if the a priori voting weights are equal, then Desideratum 3 holds if and only if at least four States participate in the voting; this is ensured by setting $\tau=1 / 10$. For modified voting games, Proposition 3 states that the condition $\alpha \geq \beta$ is ensured by choosing $\lambda \leq 1 / 3$.

\footnotetext{
2 In a previous version, we had a separate desideratum which postulated that no State should be a blocker. We thank Sascha Kurz for kindly pointing out that Desiderata 2 and 3 together imply such a postulate.
} 
So, to satisfy Desideratum 4 , it is sufficient to choose a value of $\lambda$ which is at most $1 / 3$.

Remark The present GST rules permit the possibility of strategic voting, either by being absent, or, by being present but, abstaining. This may not be desirable. Nonetheless, this feature may also not be avoidable. The members of the GST Council are elected representatives. It is perhaps difficult to pass a law whereby elected representatives are forced to be present in a meeting and to take part in the voting. The desiderata that we have formulated do not address the issue of strategic voting. Preventing strategic voting will require a much more drastic change to the GST voting rule than what we have proposed. We leave it to future work to come up with such a change of rules and associated socio-political justifications.

\section{Conclusion}

This work carried out an in-depth theoretical analysis of the voting rule specified in the GST Act. The analysis has been done using the formal framework of voting games. We have identified a set of desiderata and put forward suggestions for modifying the voting rule in the GST Act.

Acknowledgements We are indebted to Satya R. Chakravarty, Sascha Kurz and Stefan Napel for providing comments on an earlier version which have helped in improving the paper. Thanks to the reviewers for suggestions which have helped in improving the paper.

Open Access This article is licensed under a Creative Commons Attribution 4.0 International License, which permits use, sharing, adaptation, distribution and reproduction in any medium or format, as long as you give appropriate credit to the original author(s) and the source, provide a link to the Creative Commons licence, and indicate if changes were made. The images or other third party material in this article are included in the article's Creative Commons licence, unless indicated otherwise in a credit line to the material. If material is not included in the article's Creative Commons licence and your intended use is not permitted by statutory regulation or exceeds the permitted use, you will need to obtain permission directly from the copyright holder. To view a copy of this licence, visit http://creativecommons.org/licenses/by/4.0/.

\section{Appendix: Proofs}

Proof of Theorem 1 Note that $\omega=w_{0}+w_{1}+\cdots+w_{n}=u b / d$ and so $w_{0} / \omega=a / b=$ $\lambda$ and $w_{i} / \omega=u_{i}(b-a) /(u b)=(1-a / b) u_{i} / u=(1-\lambda) u_{i} / u$. This shows that $w_{0}, w_{1}, \ldots, w_{n}$ determined by (3) satisfy (4). 
The following computation shows the second part of the result.

$$
\begin{aligned}
{\left[w_{0}^{\prime}: w_{1}^{\prime}: \cdots: w_{n}^{\prime}\right] } & =\left[\frac{w_{0}^{\prime}}{\omega^{\prime}}: \frac{w_{1}^{\prime}}{\omega^{\prime}}: \cdots: \frac{w_{n}^{\prime}}{\omega^{\prime}}\right] \\
& =\left[\lambda:(1-\lambda) \frac{u_{1}}{u}: \cdots:(1-\lambda) \frac{u_{n}}{u}\right] \\
& =\left[\frac{a}{b}: \frac{(b-a) u_{1}}{b u}: \cdots: \frac{(b-a) u_{n}}{b u}\right] \\
& =\left[a u:(b-a) u_{1}: \cdots:(b-a) u_{n}\right] \\
& =\left[\frac{a u}{d}: \frac{(b-a) u_{1}}{d}: \cdots: \frac{(b-a) u_{n}}{d}\right] \\
& =\left[w_{0}: w_{1}: \cdots: w_{n}\right] .
\end{aligned}
$$

Proof of Proposition 1 From (3), we have $w_{i}<w_{0}$ if and only if $u_{i} / u<a /(b-a)$ where $u=u_{1}+\cdots+u_{n}$. Using $\lambda=a / b$ we obtain the required result.

Proof of Proposition 2 By the definition, $\lambda<q$ and so the primary player is not a dictator and consequently, it can be argued that $\alpha, \beta>0$.

Let the weight of the primary player be $w_{0}$ and that of the $n$ secondary players be $w_{1}, \ldots, w_{n}$ where we assume without loss of generality that $w_{1} \geq w_{2} \geq \cdots \geq w_{n}$. Let $\omega=w_{0}+w_{1}+\cdots+w_{n}$. Note that $w_{0} / \omega=\lambda$. Since $G$ is $(\alpha, \beta)$-efficient with respect to the primary player, it follows that a minimum size winning coalition contains $\alpha$ secondary players and a minimum size blocking coalition consisting only of secondary players has $\beta$ players. Using the order condition on the $w_{i}$ 's, we have $w_{0}+w_{1}+\cdots+w_{\alpha} \geq q \omega$ and $w_{1}+\cdots+w_{\beta}>(1-q) \omega$. Now $\alpha<\beta$ if and only if a coalition of $\alpha$ secondary players do not form a blocking coalition. The last condition holds if and only if the sum of the first $\alpha$ weights is at most $(1-q) \omega$, i.e., if and only if $w_{1}+\cdots+w_{\alpha} \leq(1-q) \omega$. Combining this with $w_{0}+w_{1}+\cdots+w_{\alpha} \geq q \omega$, we have $q \omega-w_{0} \leq w_{1}+\cdots+w_{\alpha} \leq(1-q) \omega$. So, $q \omega-w_{0} \leq(1-q) \omega$ which is equivalent to $2 q-1 \leq w_{0} / \omega=\lambda$. Rearranging, we obtain the desired inequality.

Proof of Theorem 2 Let $G_{m, n, \mathbf{v}, V, q, \lambda, \tau}$ be a uniform game where the $u_{i}$ 's are all equal. The weight $w_{0}$ of the primary player and the weights $w_{1}, \ldots, w_{n}$ of the $n$ secondary players which participate in the voting are obtained from (3). Since the $u_{i}$ 's are equal, so are the $w_{i}$ 's. Let $\omega=w_{0}+w_{1}+\cdots+w_{n}$. So, $w_{0}=\lambda \omega$ and $w_{i}=(1-\lambda) \omega / n$ for $i=1, \ldots, n$.

Now consider a game $G_{m, n, \mathbf{v}^{\prime}, V, q, \lambda, \tau}^{\prime}$ where the a priori weights $v_{i}^{\prime}$ of the secondary players are not equal. The weights in the voting game are again obtained from (3). In $G^{\prime}$, let the weight of the primary player be $w_{0}^{\prime}$ and the weights of the secondary players be $w_{1}^{\prime}, \ldots, w_{n}^{\prime}$. Without loss of generality, we assume that $w_{1}^{\prime} \geq w_{2}^{\prime} \geq \cdots \geq w_{n}^{\prime}$. Let $\omega^{\prime}=w_{0}^{\prime}+w_{1}^{\prime}+\cdots+w_{n}^{\prime}$. So, $w_{0}^{\prime}=\lambda \omega^{\prime}$ and $w_{1}^{\prime}+\cdots+w_{n}^{\prime}=(1-\lambda) \omega^{\prime}$.

Claim 1 The minimum of the sizes of the minimal winning coalitions in $G^{\prime}$ is at most the minimum of the sizes of the minimal winning coalitions in $G$. 
Proof of Claim 1 Suppose $G$ has a winning coalition consisting of the primary player and $k$ of the secondary players. Then

$$
\frac{\lambda \omega+k \omega(1-\lambda) / n}{\omega}=\lambda+\frac{k(1-\lambda)}{n} \geq q .
$$

In $G^{\prime}$, consider the coalition $S$ consisting of the primary player and the secondary players having weights $w_{1}^{\prime}, \ldots, w_{k}^{\prime}$. We show that $S$ is a winning coalition.

$$
\frac{w_{S}}{\omega^{\prime}}=\frac{w_{0}^{\prime}+w_{1}^{\prime}+\cdots+w_{k}^{\prime}}{\omega^{\prime}}=\lambda+(1-\lambda) \frac{w_{1}^{\prime}+\cdots+w_{k}^{\prime}}{w_{1}^{\prime}+\cdots+w_{n}^{\prime}} .
$$

From $w_{1}^{\prime} \geq w_{2}^{\prime} \geq \cdots \geq w_{n}^{\prime}$, we have

$$
(n-k)\left(w_{1}^{\prime}+\cdots+w_{k}^{\prime}\right) \geq(n-k) k w_{k}^{\prime} \geq k(n-k) w_{k+1}^{\prime} \geq k\left(w_{k+1}^{\prime}+\cdots+w_{1}^{\prime}\right) .
$$

Adding $k\left(w_{1}^{\prime}+\cdots+w_{k}^{\prime}\right)$ to both sides, we have $n\left(w_{1}^{\prime}+\cdots+w_{k}^{\prime}\right) \geq k\left(w_{1}^{\prime}+\cdots+w_{n}^{\prime}\right)$ and consequently

$$
\frac{w_{1}^{\prime}+\cdots+w_{k}^{\prime}}{w_{1}^{\prime}+\cdots+w_{n}^{\prime}} \geq \frac{k}{n}
$$

Combining (8) with (7) and (9) we obtain

$$
\frac{w_{S}}{\omega^{\prime}}=\lambda+(1-\lambda) \frac{w_{1}^{\prime}+\cdots+w_{k}^{\prime}}{w_{1}^{\prime}+\cdots+w_{n}^{\prime}} \geq \lambda+\frac{k(1-\lambda)}{n} \geq q .
$$

This shows that if there is a winning coalition of size $k$ in $G$, then there is also a winning coalition of size $k$ in $G^{\prime}$. So, the minimum of the sizes of the minimal winning coalitions in $G^{\prime}$ is at most the minimum of the sizes of the minimal winning coalitions in $G$. This establishes Claim 1.

Claim 2 The minimum of the sizes of the minimal blocking coalitions not containing the primary player in $G^{\prime}$ is at most the minimum of the sizes of the minimal blocking coalitions not containing the primary player in $G$.

Proof of Claim 2 In $G$, suppose that $\ell$ of the secondary players form a blocking coalition. Then

$$
\frac{\ell(1-\lambda) \omega / n}{\omega}=\frac{\ell(1-\lambda)}{n}>1-q .
$$

In $G^{\prime}$, consider the coalition $T$ consisting of the secondary players having the weights $w_{1}^{\prime}, \ldots, w_{\ell}^{\prime}$. Then

$$
\frac{w_{T}}{\omega^{\prime}}=\frac{w_{1}^{\prime}+\cdots+w_{\ell}^{\prime}}{\omega^{\prime}}=(1-\lambda) \frac{w_{1}^{\prime}+\cdots+w_{\ell}^{\prime}}{w_{1}^{\prime}+\cdots+w_{n}^{\prime}} .
$$


As in the proof of Claim 1, it can be shown that $\frac{w_{1}^{\prime}+\cdots+w_{\ell}^{\prime}}{w_{1}^{\prime}+\cdots+w_{n}^{\prime}} \geq \frac{\ell}{n}$, and so from (11) and (10), we obtain $\frac{w_{T}}{\omega^{\prime}} \geq \lambda+\frac{\ell(1-\lambda)}{n}>1-q$. This shows that if there is a blocking coalition not containing the primary player of size $\ell$ in $G$, then there is a blocking coalition not containing the primary player of size $\ell$ in $G^{\prime}$. So, the minimum of the sizes of the minimal blocking coalitions not containing the primary player in $G^{\prime}$ is at most the minimum of the sizes of the minimal blocking coalitions not containing the primary player in $G$. This establishes Claim 2 .

From Claims 1 and 2, it follows that if $G$ is $(\alpha, \beta)$-efficient with respect to the primary player, then $G^{\prime}$ is $\left(\alpha^{\prime}, \beta^{\prime}\right)$-efficient with respect to the primary player where $\alpha^{\prime} \leq \alpha$ and $\beta^{\prime} \leq \beta$.

Proof of Theorem 3 Let $\lambda=a / b$. Since all the a priori weights are equal, from (3) we have $u=n, w_{0}=a n / d$ and $w_{i}=(b-a) / d$ for $i=1, \ldots, n$ and so $\omega=b u / d=$ $b n / d$. Suppose the primary player and $k$ other secondary players form a coalition. Such a coalition is a winning coalition if and only if

$$
\begin{aligned}
\frac{k(b-a)}{d}+ & \frac{a n}{d} \geq q \frac{b n}{d} \\
& \Leftrightarrow k \geq \frac{n(q b-a)}{b-a}=n \frac{q-\lambda}{1-\lambda}=n \frac{(1-\lambda)-(1-q)}{1-\lambda}=n-\frac{n(1-q)}{1-\lambda} \\
& \Leftrightarrow k \geq n-\left\lfloor\frac{n(1-q)}{1-\lambda}\right\rfloor=n-\rho \\
& \Leftrightarrow k \geq \mathfrak{p} .
\end{aligned}
$$

A coalition is blocking if and only if the sum of the weights of the players in the coalition is greater than $(1-q) \omega$. A coalition consisting of $\ell$ secondary players has weight $\ell(b-a) / d$ and so it is blocking if and only if

$$
\begin{aligned}
\frac{\ell(b-a)}{d}>\frac{(1-q) b n}{d} \\
\Leftrightarrow \ell>\frac{n(1-q) b}{(b-a)}=\frac{n(1-q)}{1-\lambda} \\
\Leftrightarrow \ell>\left\lfloor\frac{n(1-q)}{1-\lambda}\right\rfloor \\
\Leftrightarrow \ell \geq 1+\left\lfloor\frac{n(1-q)}{1-\lambda}\right\rfloor=1+\rho \\
\Leftrightarrow \ell \geq \mathfrak{q} .
\end{aligned}
$$

Proof of Theorem 5 From Theorem 3, we have $\mathfrak{p}_{1}=n-\rho_{1}$ and $\mathfrak{p}_{2}=n-\rho_{2}$ where

$$
\rho_{1}=\left\lfloor\frac{n(1-q)}{\left(1-\lambda_{1}\right)}\right\rfloor \text { and } \rho_{2}=\left\lfloor\frac{n(1-q)}{\left(1-\lambda_{2}\right)}\right\rfloor .
$$


So, $\lambda_{1} \geq \lambda_{2} \Leftrightarrow \rho_{1} \geq \rho_{2} \quad \Leftrightarrow \quad \mathfrak{p}_{1} \leq \mathfrak{p}_{2}$. The inequality cannot be made strict since it is possible that $\lambda_{1}>\lambda_{2}$ but, $\rho_{1}=\rho_{2}$.

From Theorem 3, we have $\mathfrak{q}_{1}=1+\rho_{1}$ and $\mathfrak{p}_{2}=1+\rho_{2}$. So, $\lambda_{1} \geq \lambda_{2} \Leftrightarrow \rho_{1} \geq$ $\rho_{2} \Leftrightarrow \mathfrak{q}_{1} \geq \mathfrak{q}_{2}$. Again, the inequality cannot be made strict.

\section{References}

Banzhaf JF (1965) Weighted voting doesn't work: a mathematical analysis. Rutgers Law Rev 19:317-343 Chakravarty SR, Mitra M, Sarkar P (2015) A course on cooperative game theory. Cambridge University Press, Cambridge

Felsenthal DS, Machover M (1998) The measurement of voting power. Edward Elgar, Cheltenham

Freixas J, Zwicker WS (2003) Weighted voting, abstention and multiple levels of approval. Soc Choice Welf 21:399-431

Freixas J, Zwicker WS (2009) Anonymous yes-no voting with abstention and multiple levels of approval. Games Econ Behav 67:428-444

Kurz S, Maaser N, Napel S, Weber M (2015) Mostly sunny: a forecast of tomorrow's power index research. Homo Oeconomicus 32:133-146

Laruelle A, Valenciano F (2011) Voting and collective decision-making. Cambridge University Press, Cambridge

Laruelle A, Valenciano F (2012) Quaternary dichotomous voting rules. Social Choice Welf 38(3):431-454

Leech D (2002a) Designing the voting system for the Council of the European Union. Public Choice 113:437-464

Leech D (2002b) Power in the governance of the International Monetary Fund. Ann Oper Res 109(14):375-397

Napel S (2016) Voting power. In: Congleton R, Grofman B, Voigt S (eds) Oxford handbook of public choice. Oxford University Press, Oxford

Penrose LS (1946) The elementary statistics of majority voting. J R Stat Soc 109:53-57

Shapley LS (1953) A value for $n$-person games. In: Kuhn HW, Tucker AW (eds) Contributions to the theory of games II (Annals of Mathematics Studies). Princeton University Press, Princeton, pp 307-317

Shapley LS, Shubik MJ (1954) A method for evaluating the distribution of power in a committee system. Am Polit Sci Rev 48:787-792

Publisher's Note Springer Nature remains neutral with regard to jurisdictional claims in published maps and institutional affiliations. 